\title{
The CRISPR/Cas Genome-Editing Tool: Application in Improvement of Crops
}

\author{
Surender Khatodia' ${ }^{1}$ Kirti Bhatotia' ${ }^{1}$, Nishat Passricha ${ }^{2}$, S. M. P. Khurana ${ }^{1}$ and \\ Narendra Tuteja ${ }^{2,3 *}$
}

${ }^{1}$ Amity Institute of Biotechnology, Amity University Haryana, Gurgaon, India, ${ }^{2}$ Plant Molecular Biology Group, International Centre for Genetic Engineering and Biotechnology, New Delhi, India, ${ }^{3}$ Amity Institute of Microbial Technology, Amity University, Noida, India

\section{OPEN ACCESS}

Edited by: Richard Sayre,

New Mexico Consortium at Los Alamos National Labs, USA

Reviewed by:

Vinay Kumar

Central University of Punjab, India Upinder S. Gill,

The Samuel Roberts Noble Foundation, USA

${ }^{*}$ Correspondence:

Narendra Tuteja ntuteja@amity.edu

Specialty section:

This article was submitted to

Plant Biotechnology,

a section of the journal

Frontiers in Plant Science

Received: 21 January 2015 Accepted: 30 March 2016 Published: 19 April 2016

Citation:

Khatodia S, Bhatotia K, Passricha N, Khurana SMP and Tuteja N (2016) The CRISPR/Cas Genome-Editing Tool: Application in Improvement of Crops. Front. Plant Sci. 7:506. doi: 10.3389/fpls.2016.00506
The Clustered Regularly Interspaced Short Palindromic Repeats associated Cas9/sgRNA system is a novel targeted genome-editing technique derived from bacterial immune system. It is an inexpensive, easy, most user friendly and rapidly adopted genome editing tool transforming to revolutionary paradigm. This technique enables precise genomic modifications in many different organisms and tissues. Cas9 protein is an RNA guided endonuclease utilized for creating targeted double-stranded breaks with only a short RNA sequence to confer recognition of the target in animals and plants. Development of genetically edited (GE) crops similar to those developed by conventional or mutation breeding using this potential technique makes it a promising and extremely versatile tool for providing sustainable productive agriculture for better feeding of rapidly growing population in a changing climate. The emerging areas of research for the genome editing in plants include interrogating gene function, rewiring the regulatory signaling networks and sgRNA library for high-throughput loss-of-function screening. In this review, we have described the broad applicability of the Cas 9 nuclease mediated targeted plant genome editing for development of designer crops. The regulatory uncertainty and social acceptance of plant breeding by Cas 9 genome editing have also been described. With this powerful and innovative technique the designer GE non-GM plants could further advance climate resilient and sustainable agriculture in the future and maximizing yield by combating abiotic and biotic stresses.

Keywords: CRISPR, Cas9/sgRNA, plant genome editing, GE crops, dCas9, RGENs, NPBTs

The targeted plant genome editing using sequence specific nucleases has a great potential for crop improvement to meet the increasing global food demands and to provide sustainable productive agriculture system (Liu et al., 2013). Traditionally, the crops were being improved by the conventional and mutation plant breeding techniques, which are now getting constrained by the declining of existing genetic variation of plants, hampering the production for future feeding (Chen and Gao, 2014). There is an urgent need for efficient crop improvement strategies with novel genome editing techniques like CRISPR/Cas9 system, which can improve the existing important functions or make new valuable products (Zhang and Zhou, 2014). In mere 3 years, this technique has been trending vast with numerous examples of targeted mutagenesis and targeted regulation of transcriptional control in model as well as crop plants which clearly demonstrates the revolutionary aspects of this novel system (Khatodia and Khurana, 2014). In this review, we have summarized the 
broad applicability of Cas9 nuclease mediated targeted genome editing of plants for development of designer crops. The Cas 9 system has been used extensively for gene knockouts, gene replacement, multiplex editing, interrogating gene function, and transcription modulation in animals and plants. The opportunities of the gene editing with Cas9/sgRNA for obtaining climate resilient and bio-energy agriculture and to develop nonGM plants along with regulating the uncertainty and the social acceptance of this new plant breeding technique have been prospected in this article.

\section{CRISPR/Cas TECHNOLOGY - AN OVERVIEW}

Clustered regularly interspaced short palindromic repeat/Cas system was discovered in bacteria as an adaptive immune system which helps the bacteria in protecting itself against invading foreign DNA, such as that of a bacteriophage. This system comprises of CRISPR loci in the genome and a Cas9 protein. CRISPR, i.e., Clustered Regularly Interspaced Short Palindromic Repeats (CRISPRs) - is a genomic locus of tandem direct repeat sequences and protospacers, the spaces in between repeat sequences, both of which are derived from the invading elements (Kim and Kim, 2014). The CRISPR loci contains a combination of Cas9 genes; sequences for non-coding RNA elements called CRISPR RNA (crRNA) and sequences for small trans-encoded CRISPR RNA, i.e., trans-activating crRNA (tracrRNA). The two RNA sequences crRNA and tracrRNA forms a complex known as guide RNA, which determines the specificity of the cleavage of the target sequence in the nucleic acid along with the Protosapcer Adjacent Motif (PAM), a 5'-NGG sequence (Barrangou, 2013; Jinek et al., 2013). The cleavage of the double stranded target DNA occurs within the limits of protospacer region. The Cas 9 protein is an endonuclease associated with CRISPR loci, which is responsible for the double-stranded breaks (DSBs) at the site, when targeted by a guide RNA (Cong et al., 2013; Mali et al., 2013). This revealing of molecular mechanism of the CRISPR/Cas system in 2012 opened up its vast area of applications as a promising component of genome editing termed as RNA-guided engineered nucleases (RGENs), which were used as sequence specific nucleases for precise genetic modifications (Doudna and Charpentier, 2014; Fichtner et al., 2014; Liang et al., 2015). RGENs are developed as programmable nucleases composed of two components, which must be expressed in cells to perform genome editing; the Cas9 nuclease and an engineered single guide RNA (sgRNA). The sgRNA has 20 nucleotides at the $5^{\prime}$ end that directs Cas9 to the complementary target site. Any DNA sequence of the form N20-NGG can be targeted by altering the first 20 nucleotides of the gRNA for novel genome editing applications (Sander and Joung, 2014) (Figure 1).

The targeted genome editing is utilized for the generation of desired endogenous modifications like gene disruption, addition, or correction at one or more specific genome site by introduction of nuclease mediated DNA-break using customized engineered nucleases (Kim and Kim, 2014). After DSB the cellular recombination repair mechanism can do the desired modification in a broad range of organisms and cell types. The elucidation of the CRISPR-Cas9 mechanism have resulted in many fundamental discoveries in biology (Doudna and Charpentier, 2014). CRISPR/Cas genome editing as a fledgling technology has reinvented the genetic and molecular biology research due to its simplicity and ease of design. There are many Cas9 and gRNA variants available, which could be utilized for further novel applications particularly in the field of plant biotechnology.

\section{THE Cas9 NUCLEASE VARIANTS}

The Cas9 endonuclease consists of two different domains, which includes a large globular recognition (REC) specific functional domain, connected to a smaller nuclease (NUC) domain. The NUC domain further accommodates two nuclease sites, RuvC and $\mathrm{HNH}$, and also a PAM-interacting site (Doudna and Charpentier, 2014; Jinek et al., 2014). Cas9 protein is activated upon the loading of guide RNA, which further undergoes a conformational rearrangement to form a central channel for RNA-DNA heteroduplex binding and canonical PAM motifs recognition (Anders et al., 2014). The mechanism of Cas9 nuclease action was recently elucidated by crystal structure studies in a complex with partially duplexed target DNA containing PAM motif and sgRNA. This provides an insight into how Cas9 may be engineered to create variants with novel PAM specificities (Belhaj et al., 2013). There are many Cas9 variants available today, which can be utilized for highthroughput genome editing, silencing and transcriptional control with improved specificity and reduced off target effects in various systems, from yeast, Drosophila, bacteria, monkey, zebrafish, human, and plants.

\section{The Native Cas9}

The DSB created by a native Cas9 can be repaired by either HR or NHEJ method (Wyman and Kanaar, 2006; Shuman and Glickman, 2007). HR-mediated repair can be used to introduce specific point mutations like nucleotide substitutions or to insert desired sequences through recombination of the target locus with exogenously supplied DNA templates (Jiang et al., 2013) (Figure 1). NHEJ can, however, lead to the efficient introduction of insertion/deletion mutations (indels), which can disrupt the translational reading frame of a coding sequence or the transcription factors binding sites in promoters or enhancers (Cong et al., 2013). The high rate of alterations after DSBs created by Cas9, makes easy identification of the desired mutations without drug-resistance marker selection (Shimatani et al., 2015). So far, the applicability of the native Cas9/sgRNA system has been demonstrated in 10 plant species including model crops for targeted mutagenesis to gene knockouts and replacement as well as multiplex plant gene editing (Table 1).

\section{The Cas9 Nickase}

A Cas9 nickase (Cas9n) was first developed by Cong et al. (2013) through a mutation in native Cas9 (D10A, aspartate to 


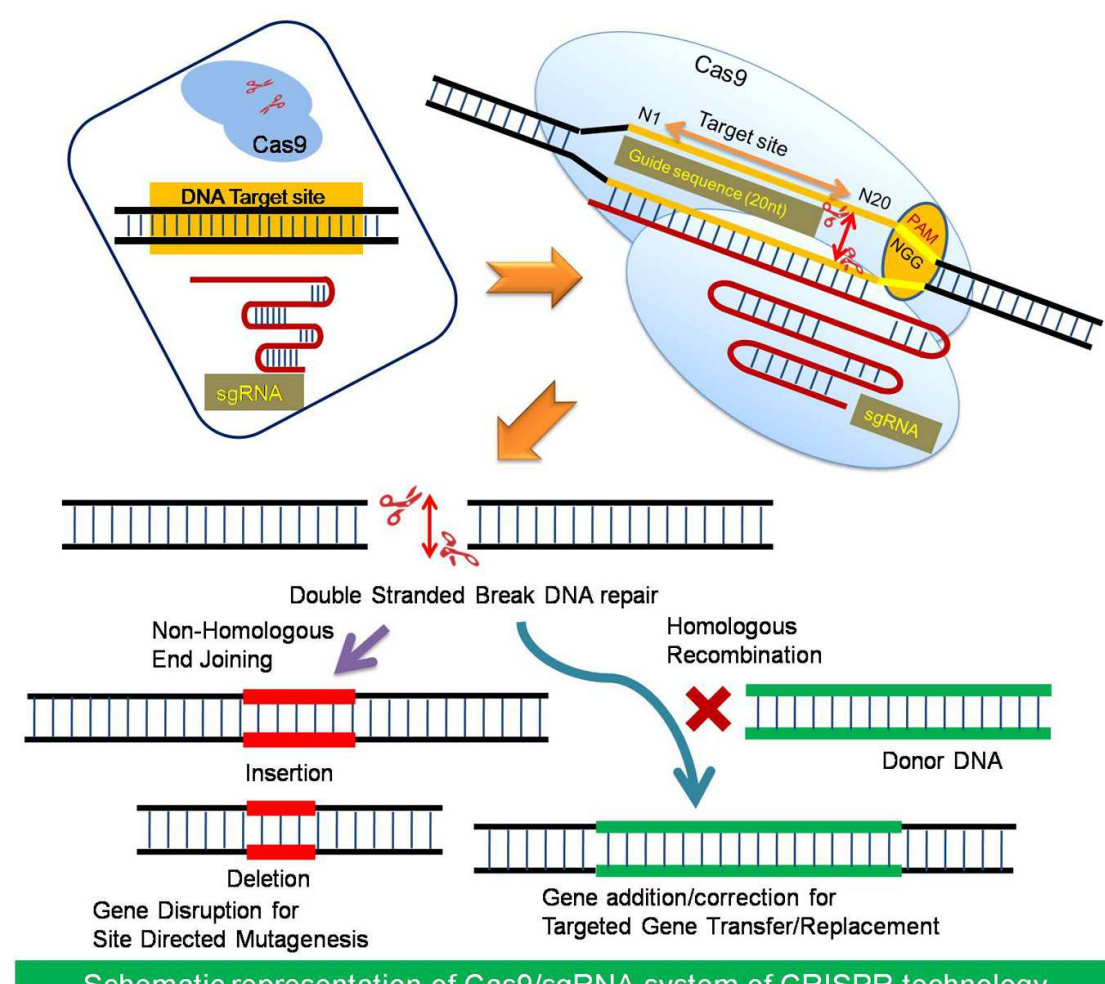

Schematic representation of Cas9/sgRNA system of CRISPR technology

FIGURE 1 | The basic strategy of Cas9/sgRNA system. The Cas9 is a RNA guided endonuclease consists of two nuclease domains namely HNH and RuvC. The target specificity of Cas9 depends upon the guide sequence (20 nt) short guide RNA (sgRNA). The target sites must lay immediately $5^{\prime}$ of a PAM (Protospacer Adjacent Motif) sequence of the form N20-NGG (or N2O-NAG). The Cas9 nuclease induces double stranded breaks (DSB) at the target site which can be repaired either by Non- Homologous End Joining method or Homologous Recombination by cellular system which results in gene disruption by indels or gene addition/correction, respectively.

alanine substitution). The Cas9 nickase with a RuvC or $\mathrm{HNH}$ mutation has the ability to create a nick, instead of a DSB at the target site. The individual nicks in the genome can be typically repaired with high fidelity homology-directed repair (HDR). The Cas9n has been used into a paired nickase system with two different gRNA to extend the number of specifically recognized bases for target cleavage, which has improved the specificity and helped mitigate the off-target phenomena (Cong et al., 2013; Mali et al., 2013; Ran et al., 2013; Fauser et al., 2014). The paired-nicking strategy has high-efficiency of HDR with reduced off-target cleavages by 50 - to 1,500-fold in human cells, without sacrificing on-target cleavage efficiency (Ran et al., 2013) (Figure 2A).

\section{The Inactive dCas9}

The nuclease deficient catalytically inactive mutant version of Cas9 (dCas9) has been used for RNA-guided transcription regulation, instead of genome editing (Gilbert et al., 2013; Qi et al., 2013). This modified system has been used for CRISPR interference (CRISPRi) and CRISPR activator (CRISPRa) for highly efficient and precise gene silencing and activation, respectively, using dCas 9 with an effector and sgRNA. The dCas9 has an ability to incorporate gRNA and binding to the target ( $\mathrm{Xu}$ et al., 2014). In comparison to the
RNAi concept of transcript degradation and/or translation blocking, the CRISPRi system blocks the transcription initiation and elongation when dCas9/sgRNA is fused with a repressor. Therefore, dCas9/sgRNA system offers a general platform for RNA-guided DNA targeting for stable and efficient modulation of transcription. The dCas9 has been fused to effector domains with distinct regulatory functions for functional mapping of promoters and other genomic regulatory modules (Gilbert et al., 2013; Qi et al., 2013; Figure 3).

Lowder et al. (2015) developed a CRISPR/Cas9 toolbox of applications in plants by transcriptional regulation. They have examined the applicability of the fusing the deactivated Cas9 with the transcriptional activator VP64 or the transcriptional repressor domain SRDX for transcriptional activation and repression of multiple endogenous genes (Lowder et al., 2015). The dCas9 has also been used to deliver GFP to targeted genomic locations (Anton et al., 2014). The novel light and chemical inducible dCas9 system have been developed, which could be utilized for light and agrochemical mediated transcription activation using Cas9 system. These will further, widen the applicability of Cas9/sgRNA system in crop improvement and functional genomics (Cong et al., 2013; Polstein and Gersbach, 2015; Zetsche et al., 2015). 
TABLE 1 | List of applications of Cas9/sgRNA system in various plant species for single or multiplex genome editing and gene insertion or replacement.

\begin{tabular}{|c|c|c|}
\hline Plant species & Target genes & Reference \\
\hline \multicolumn{3}{|c|}{ Gene knockout or editing with Cas9/sgRNA } \\
\hline Arabidopsis thaliana & $\begin{array}{l}\text { AtPDS3, AtFLS2, TT4,BRI1, JAZ1, GAl,ADH1, CHLI, AP1, FT, } \\
\text { SPL4, AtCRU3, At1g56650 }\end{array}$ & $\begin{array}{l}\text { Feng et al., 2013; Jiang et al., 2013; Li et al., 2013; Mao et al., 2013; } \\
\text { Fauser et al., 2014; Feng et al., 2014; Jiang et al., 2014; Hyun et al., 2015; } \\
\text { Johnson et al., 2015; Ma et al., } 2015\end{array}$ \\
\hline Nicotiana benthamiana & NbPDS, PDS, NbPDS, NbPDS3, NblspH & $\begin{array}{l}\text { Jiang et al., 2013; Li et al., 2013; Nekrasov et al., 2013; Upadhyay et al., } \\
\text { 2013; Yin et al., } 2015\end{array}$ \\
\hline Nicotiana tabacum & NtPDS, NtPDR6, ALS & Baltes et al., 2014; Gao et al., 2014 \\
\hline Oryza sativa & $\begin{array}{l}\text { OsPDS,OsBADH2,Os02g23823,OsMPK2, OsSWEET11, } \\
\text { OsSWEET14, CAO1, LAZY1, OsMPK5, OsMYB1, ROC5, SPP, } \\
\text { YSA.BEL,SWEET13/1a/1b, PMS3, EPSPS, DERF1, MSH1, } \\
\text { MYB5, CDKB2, OsGSTU, OsMRP15, OsAnP, OsAOX1a, } \\
\text { OsAOX1b, OsAOX1c, OsBEL }\end{array}$ & $\begin{array}{l}\text { Feng et al., 2013; Jiang et al., 2013; Mao et al., 2013; Miao et al., 2013; } \\
\text { Shan et al., 2013; Xie and Yang, 2013; Endo et al., 2014; Xu et al., 2014, } \\
\text { 2015; Zhang et al., 2014; Zhou et al., 2014; }\end{array}$ \\
\hline Triticum Aestivum & TaMLO,INOX, PDS, TaMLO-A1 & Shan et al., 2013; Upadhyay et al., 2013; Wang et al., 2014 \\
\hline Sorghum bicolor & DsRED2 & Jiang et al., 2013 \\
\hline Marchantia polymorpha & MpARF1 & Sugano et al., 2014 \\
\hline Citrus sinensis & CSPDS & Jia and Wang, 2014 \\
\hline Solanum lycopersicum & SIAGO7,mGFP5, eGFP, RIN, ANT1 & Brooks et al., 2014; Ron et al., 2014; Cermak et al., 2015; Ito et al., 2015 \\
\hline Zea mays & ZmIPK, LIG1, Ms26, Ms45, ALS2 & Liang et al., 2014; Svitashev et al., 2015 \\
\hline Glycine max & $\begin{array}{l}\text { Glyma07g14530, Glyma06g14180, Glyma08g02290, } \\
\text { Glyma12g37050, Glyma18g04660, Glyma20g38560; GmFEl2, } \\
\text { GmSHR }\end{array}$ & Cai et al., 2015; Jacobs et al., 2015; Michno et al., 2015; Sun et al., 2015 \\
\hline Medicago trancatula & GUS & Michno et al., 2015 \\
\hline Populus tomentosa & PtoPDS, 4CL, PtPDS & Fan et al., 2015; Tingting et al., 2015; Zhou et al., 2015 \\
\hline Solanum tubersum & StIAA2, StALS1 & Butler et al., 2015; Wang et al., 2015 \\
\hline \multicolumn{3}{|c|}{ Gene knockout or editing with Cas9 paired nickase/sgRNA } \\
\hline Arabidopsis thaliana & RTEL1, ADH1, TT4 & Fauser et al., 2014; Schiml et al., 2014 \\
\hline \multicolumn{3}{|c|}{ Multiplex genome editing with Cas9/sgRNA } \\
\hline Arabidopsis thaliana & $\begin{array}{l}\text { AtRACK1b+AtRACK1C, CHL11+CHL12, ETC2, CPC, TRY, and } \\
\text { PYL1-6, At5g55580 }\end{array}$ & $\begin{array}{l}\text { Li et al., 2013; Mao et al., 2013; Xing et al., 2014; Ma et al., 2015; Zhang } \\
\text { et al., } 2015\end{array}$ \\
\hline Nicotiana tabacum & $N t P D S+N t P D R 6$ & Gao et al., 2014 \\
\hline Oryza sativa & CDKB1, CDKA1, MPK1/2/5/6, PDSOSFTL & Endo et al., 2014; Ma et al., 2015; Xie et al., 2015 \\
\hline Solanum lycopersicum & Solyc07g021170+Solyc12g044760 & Brooks et al., 2014 \\
\hline Zea mays & $Z m H K T 1$ & Xing et al., 2014 \\
\hline Triticum aestivum & TaMLO-A1+TaMLO-B1+TaMLO-D1 & Wang et al., 2014 \\
\hline Glycine max & 01gDDM1, 11gDDM1, 01g+11gDDM1; GmFEl2, and GmSHR & Cai et al., 2015; Jacobs et al., 2015 \\
\hline Populus tomentosa & PtPDS1 and PtPDS2 & Tingting et al., 2015 \\
\hline \multicolumn{3}{|c|}{ Gene insertion or replacement by HR with Cas9/sgRNA and donor template } \\
\hline Arabidopsis thaliana & YFFP,GU.US, DGU.US $\rightarrow$ GUS functional, nptll $\rightarrow$ ADH1 & Feng et al., 2013; Mao et al., 2013; Fauser et al., 2014; Schiml et al., 2014 \\
\hline Nicotiana benthamiana & Avrll site $\rightarrow \mathrm{NbPDS}$ & Li et al., 2013 \\
\hline Oryza sativa & OsPDS,OsBADH2 & Shan et al., 2013 \\
\hline Zea mays & UBI:MOPAT & Svitashev et al., 2015 \\
\hline
\end{tabular}

\section{Dimeric RNA-Guided FokI Nucleases (RFNs)}

Dimeric RNA-guided FokI Nucleases (RFNs) are a fusion of a catalytically inactive dCas9 protein with the FokI nuclease domain. Dimerization of two RFNs rather than co-localization is required for efficient genome editing activity, which gives a plus point over the Cas9 nickase for high genome editing frequencies and reduced off-target mutations (Tsai et al., 2014; Bortesi and Fischer, 2015). Their cleavage activity depends strictly on the binding of two gRNAs with a defined spacing and orientation, which reduces the likelihood of target site occurring more than once in the genome (Tsai et al., 2014) (Figure 2B).

\section{GUIDE RNA VARIANTS}

There are some other modifications in the guide RNA of the Cas9/sgRNA system, which have provided other improvements in the native system for utilization in broader applicability of this significant technology.

\section{Truncated Guide RNAs (truRNA)}

Truncated guide RNAs (truRNA) are the sgRNA variants with shorter regions of target complementarities that is 17 nucleotides in length, which offers a simple, effective strategy to improve the specificities of Cas 9 nucleases or paired nickases by reducing the off-target effects (Fu et al., 2014; Bortesi and Fischer, 


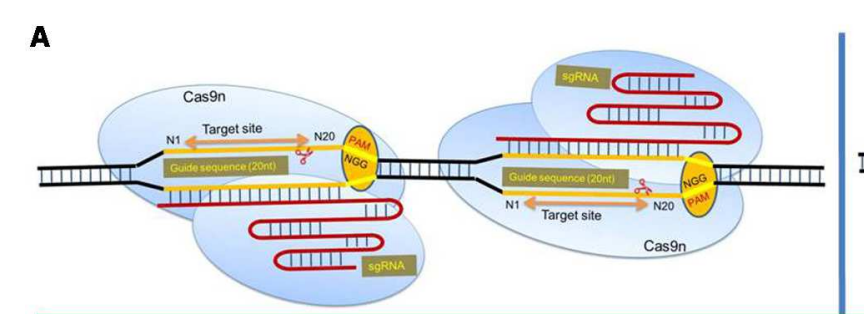

Dimeric Cas9 nickase

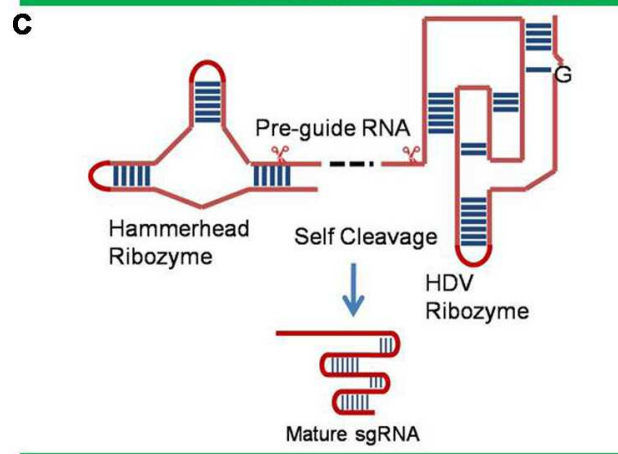

Ribozyme-gRNA-Ribozyme (RGR)

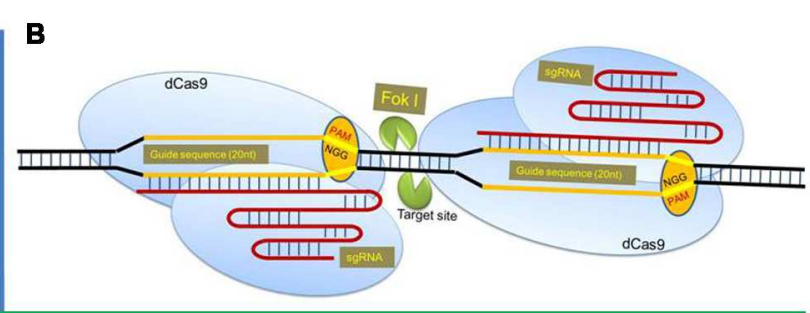

RNA-guided Fokl nucleases (RFNs)

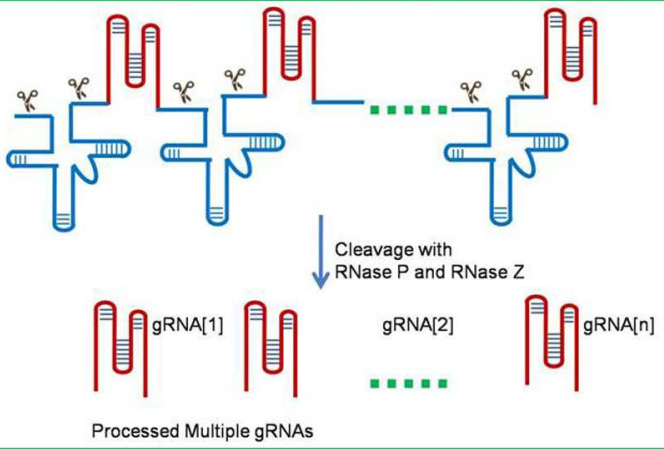

Primary Polycistronic tRNA-gRNA (PTG)

FIGURE 2 | The different variants of Cas9/sgRNA system of genome editing. (A) The Cas9 nickase (Cas9n) with a RuvC or HNH mutation create a nick instead of a DSB at the target site. The dimeric Cas9n can be used for enhances specificity and reduced off target effects. (B) Dimeric RNA-guided Fokl nucleases (RFNs) are the fusion of a catalytically inactive dCas9 protein with the Fokl nuclease domain. Dimerization of two RFNs used for high genome editing frequencies and reduced off-target mutations. (C) Ribozyme-gRNA-Ribozyme (RGR) is an artificial gene, which generates self-catalyzed desired gRNA after transcribed from any promoter for tissue-specific genome editing. (D) Primary Polycistronic tRNA-gRNA (PTG) is tandemly arrayed tRNA-gRNA units, which is cleaved by the endogenous tRNA-processing system for simultaneously targeting multiple sites.

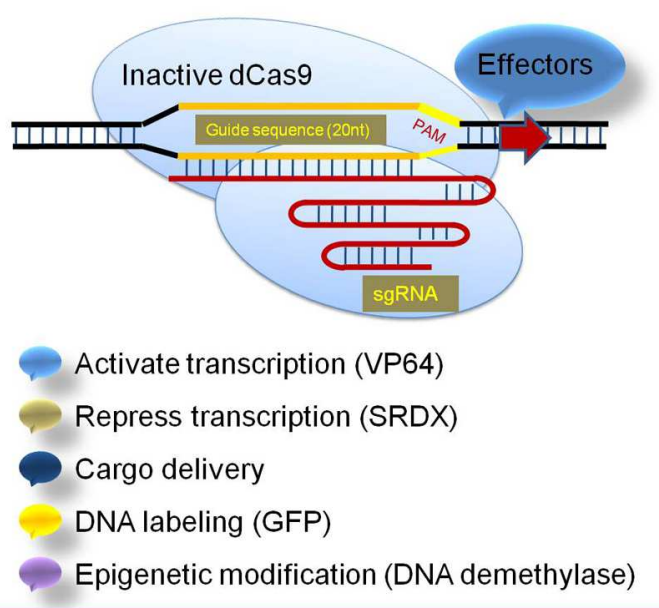

CRISPRi for probing genomic function with inactive dCas9

FIGURE 3 | The strategic demonstration of inactive Cas9 system for Regulation of transcription and effector delivery. The inactive dCas9 is a catalytically inactive mutant repurposed for RNA-guided transcription regulation for high efficiency and specific CRISPR interference (CRISPRi). It has been used by fusion of dCas9 to effector domains like activator or repressor for RNA-guided DNA modulation of transcription. The dCas9 has also been used to deliver specific effectors to targeted genomic locations like GFP and DNA demethylase.
2015). The truncated gRNAs, with shorter regions of target complementarities can decrease undesired mutagenesis at some off-target sites without sacrificing on-target genome editing efficiencies (Fu et al., 2014).

\section{Ribozyme-gRNA-Ribozyme (RGR)}

Ribozyme-gRNA-Ribozyme (RGR) is an artificial gene which generates RNA molecule with ribozyme sequences, which undergo self-catalyzed cleavage to generate the desired gRNA both in vitro and in vivo (Gao and Zhao, 2014b). RGR can be transcribed from any type of promoter and thus allow tissuespecific genome editing and efficient detection of mutations (Figure 2C).

\section{Polycistronic tRNA-gRNA (PTG/Cas9)}

Polycistronic tRNA-gRNA (PTG/Cas9) consists of array of tandem tRNA-gRNA units, with each gRNA containing a targetspecific spacer for simultaneously targeting multiple sites (Xie et al., 2015). The primary transcript of PTG is cleaved after precise processing via the endogenous tRNA-processing system by RNase $\mathrm{P}$ and RNase $\mathrm{Z}$, which releases numerous mature gRNAs in vivo from a synthetic polycistronic gene. The excised mature gRNAs direct Cas9 to multiple targets which significantly increase CRISPR/Cas9 multiplex editing efficiency in plants. Xie et al. (2015) demonstrated that targeting one gene with two gRNAs using PTG would greatly increase the efficiency 
of complete gene knock-out in comparison to the sgRNA (Figure 2D).

\section{THE Cas9/sgRNA SYSTEM FOR PLANT GENOME EDITING}

There are broadly three categories of applications of the RNA guided endonuclease particularly in plants. First, in which DSBs created by Cas9 were repaired by non-homologous end joining (NHEJ) method for generation of indels, which leads to frame-shift mutations similar to natural variants, or those produced by physical or chemical mutagenesis as in mutation breeding (Chen and Gao, 2014; Saika et al., 2014). In second category, a short DNA repair template or a transgene has been used with Cas9 to repair DSB by homologous recombination (HR) for generation of the point mutations or targeted transgene insertion, gene replacement and gene stacking at predetermined sites. This avoids the position effects associated with random insertion of genes into plant genomes using genetic engineering. Third category uses, the multiplex genome editing for targeting multiple different sites with multiple sgRNAs along with the Cas9 nuclease. The multiplex genome editing in plants can be used for dissecting the functions of gene family members with redundant functions and for analyzing epistatic relationships in genetic pathways (Xing et al., 2014). Here, we have briefly reviewed the achievements of Cas9 mediated genome editing in plants (Table 1).

\section{CRISPR Achievements in Plants}

The CRISPR/Cas system generates stable and heritable mutations, which can easily segregate from the Cas $9 / \mathrm{sgRNA}$ construct to avoid further modifications by CRISPR/Cas. This results in development of homozygous modified transgene free plants in only a generation (Brooks et al., 2014; Fauser et al., 2014; Feng et al., 2014; Gao and Zhao, 2014a; Jiang et al., 2014; Schiml et al., 2014; Zhang et al., 2014; Zhou et al., 2014). Xu et al. (2015) successfully developed transgene-free rice with desired gene mutation by segregating out the transgene with self-fertilization in the T1 generation. The relative cleavage efficiency of Cas9 nucleases has been found better in comparison to previouslydescribed TALENs and ZFNs against the same target sites (Gaj et al., 2013; Johnson et al., 2015). Xing et al. (2014) have developed a toolkit for multiplex genome editing in plants using CRISPR/Cas9-based binary vector set and a gRNA module vector set. This will facilitate transient or stable expression of CRISPR/Cas9 in a variety of plant systems and is especially useful for high-efficiency multiplex plant genome editing (Xing et al., 2014). Hence, the delivery of just two components, i.e., Cas9 and sgRNA to the host cell by genetic transformation methods is the only requirement for plant genome editing.

Baltes et al. (2014) have suggested that Gemini virus replicons (GVRs) can be used to deliver Cas9/sgRNA to plant cells with enhanced mutagenesis, in case when replication initiation protein gene (REP) was co-transformed with the Cas9/sgRNA construct. Further, to exploit the usefulness of this technology for trait discovery and development, efficient delivery methods like viruses based DNA replicons could be used for delivery of genome engineering reagents to all plant parts with higher repair frequencies and getting seeds with the desired modifications, without transformation (Baltes et al., 2014; Ali et al., 2015b). Two recent reports of direct delivery using the Tobacco rattle virus (TRV) (Ali et al., 2015b) and Cabbage Leaf Curl virus (CaLCuV) (Yin et al., 2015) have clearly demonstrated the feasibility of different virus medited Cas9/sgRNA delivery for efficient plant genome editing.

\section{CRISPRi in Plants}

The CRISPRi (CRISPR interference) has been demonstrated for RNA-guided, stable and efficient modulation of transcription of target genes in plants by fusion of inactivated dCas9 to effector domains (Larson et al., 2013). dCas9 has been used for functional genetics for regulation of gene expression and novel synthetic biology applications. It has been recruited to specific DNA sequences by gRNAs as a fusion protein with the activation or repression domain of a transcription factor (Gilbert et al., 2013; Piatek et al., 2014). The transcription of both a reporter construct and the endogenous PDS gene in Nicotiana benthamiana, have been modulated by fusing the dCas9 C-terminus to the EDLL domain as transcriptional activators, and to the SRDX domain as a repressor (Piatek et al., 2014). The recognition complex of dCas9/sgRNA/effector interferes with transcriptional regulation for efficient sgRNA dependent inducible and reversible inhibition of gene expression (Qi et al., 2013; Laganà et al., 2014). Gilbert et al. (2015) identified that the target site for effective CRISPRi should lie from -50 to +300 bp relative to the Transcription Start Site (TSS) of a gene.

CRISPR activator (CRISPRa) system modulates gene expression over a $\sim 1,000$-fold range by expression of a single sgRNA with one binding site (Gilbert et al., 2015). Development of genome-scale CRISPRi and CRISPRa libraries, would prove to be powerful tools for mapping complex stress-related signaling pathways in plants for functional genomics analysis (Laganà et al., 2014; Tingting et al., 2015; Gilbert et al., 2015). The available online resources of the CRISPR/Cas system's materials and tools have made the wide adoption and applications of this technique very simple (Table 2). These include the web resources for CRISPR/Cas system and software tools for sgRNA designing with minimized off-target effects.

\section{Virus Interference in Plants: the CRISPR Approach}

The CRISPR/Cas system of genome editing has been used as a tool for imparting resistance to viruses in plants (ChaparroGarcia et al., 2015). Three recent reports have described the CRISPR/Cas approach for protection to plants against geminiviruses (Ali et al., 2015a; Baltes et al., 2015; Ji et al., 2015). This system conferred enhanced resistance to the plants against the geminiviruses species including BCTV (Beet curly top virus), TYLCV (Tomato yellow leaf curl virus), and MeMV (Merremia mosaic virus) at the same time with single gRNA (Ali et al., 
TABLE 2 | Available online resources for CRISPR/Cas system.

\begin{tabular}{|c|c|c|}
\hline Name & Remarks & Reference \\
\hline Addgene & Reagents and resources & https://www.addgene.org/crispr/ \\
\hline sgRNA Designer & Guide RNA Design tool & http://broadinstitute.org/rnai/public/analysis-tools/sgrna-design \\
\hline Cas9 Design & Guide RNA DesignTool & http://cas9.cbi.pku.edu.cn \\
\hline $\mathrm{CHOPCHOP}$ & Target sites finding tool & https://chopchop.rc.fas.harvard.edu \\
\hline CRISPR Design & Design and analysis of Guide RNA & http://crispr.mit.edu \\
\hline CRISPR Genome Analyzer & Genome editing experiment analysis plateform & http://crispr-ga.net \\
\hline CRISPR-PLANT & Genome-wide gRNAs prediction tool in plants & http://genome.arizona.edu/crispr \\
\hline CRISPRseek & Target-specific guide RNAs design tool & http://bioconductor.org/packages/release/bioc/html/CRISPRseek.html \\
\hline DNA 2.0 gRNA Design Tool & gRNA Design tool & https://dna20.com/eCommerce/cas9/input \\
\hline E-CRISP & Target sites design tool & http://e-crisp-test.dkfz.de/E-CRISP \\
\hline RGEN Tools & Potential off-target sites prediction tool & http://rgenome.net/cas-offinder \\
\hline sgRNAcas9 & sgRNA design and potential off-target sites prediction tool & http://biootools.com \\
\hline CRISPR MultiTargeter & Multiplex design tool & http://multicrispr.net/ \\
\hline CRISPR-P & Guide RNA design in plants & http://cbi.hzau.edu.cn/crispr/ \\
\hline AGEseq & Analysis of Genome Editing by Sequencing & https://github.com/liangjiaoxue/AGEseq \\
\hline Stupar Lab's CRISPR Design & Target sites identifier & http://stuparcrispr.cfans.umn.edu/CRISPR/ \\
\hline
\end{tabular}

2015a); BeYDV (Bean yellow dwarf virus) (Baltes et al., 2015), and BSCTV (Beet severe curly top virus) (Ji et al., 2015).

\section{REGULATIONS AND SOCIAL ACCEPTANCE OF CRISPR EDITED CROPS}

Routine targeted mutagenesis in plants by the CRISPR/Cas9 system will definitely help open up new dimensions in plant biology research. The conventional random mutagenesis approaches were not able to access every gene for inactivation study because of the random nature of the gene integration. CRISPR-Cas9 technology would be of great help in generating mutants for inaccessible genes, mutate multiple loci and generate large deletions, which can, therefore, accelerate plant breeding without actually introducing a transgene (Figure 4). Although, genetically modified crops could have been a solution for crop improvement, if the controversies concerning the probable environmental and health implications of GM crops could be avoided (Hilbeck et al., 2011). Using the technique of genetic engineering a DNA construct can be directly inserted into one or more chromosomes in a random manner for manipulation of the genome. However, the random gene insertions can have undesirable effects and are not favorable for making large intensive changes, such as in case of adding an entire metabolic pathway into a plant (Lau et al., 2014). The plant breeding techniques have been used regularly to introduce new traits into cultivated varieties using existing natural genetic variation and random mutagenesis. The CRISPR/Cas system introduces

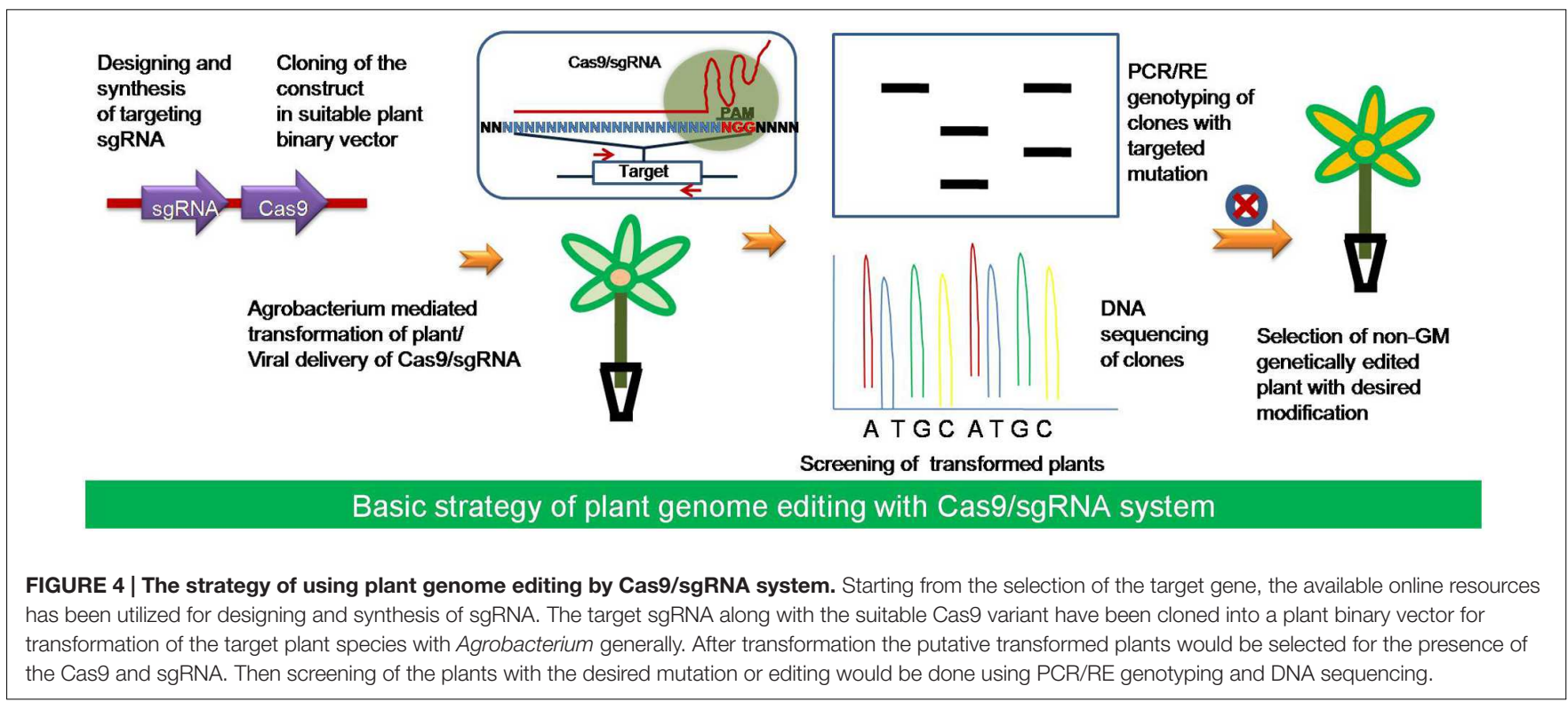


precise modifications into a plant genome, which inherits stably and transgene region could be removed simply after the target gene editing to make transgene free plants during crop variety improvement (Mahfouz et al., 2014; Gao and Zhao, 2014b; Kanchiswamy et al., 2015; Xu et al., 2015; Figure 5). The Cas9/sgRNA system, is now one of the new plant breeding techniques (NPBT) along with the ZFNs (Zinc Finger Nucleases) and TALENs (Transcription activator like effector nucleases) like genome editing technologies. The NPBTs are faster than traditional breeding methods and can produce a null segregant line that notably lacks the transgenic insert (Podevin et al., 2012; Araki and Ishii, 2015; Schaart et al., 2015; Woo et al., 2015). The plants developed by NPBTs are identical to the classically bred plants and these should be evaluated according to the resulting end product rather than the process of creation (Giddings et al., 2012; Hartung and Schiemann, 2014; Woo et al., 2015). Thus, in such a case CRISPR edited plants might be out of the current GMO regulations, but still this pose new challenges for the regulation and social acceptance of genetically edited (GE) crops (Voytas and Gao, 2014; Araki and Ishii, 2015; Jones, 2015; Kanchiswamy et al., 2015). NPBTs are currently in debate by advisory and regulatory authorities in relation to the GMO legislation to classify GE crop varieties produced using genome editing as non-GM for the social acceptance (Lusser et al., 2012; Voytas and Gao, 2014; Araki and Ishii, 2015).

Araki and Ishii (2015) suggested a regulatory concept for GE crops based on the regulatory responses from the authorities in the world by categorizing the genome edited organisms under product- based and process-based GMO regulations. They recommend that those crops which have been engineered at gene/genome level using CRISPR-Cas9 technology should not be considered GMO under product based GMO regulation, because the final products are not genetically modified organism. While more work is needed to optimize the CRISPR/Cas

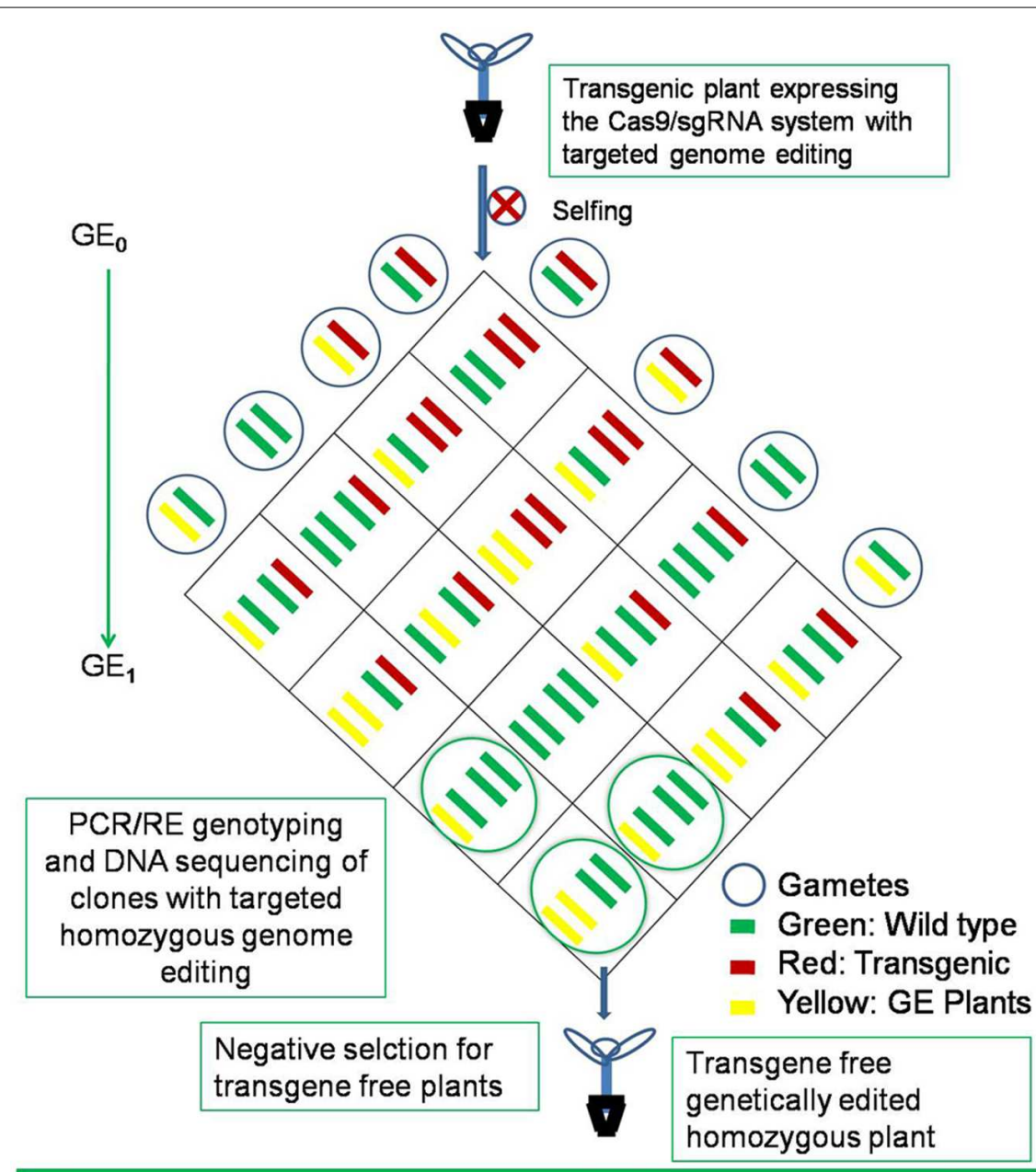

Generation of transgene free genetically edited plants with Cas9

FIGURE 5 | The generation of transgene free genetically edited (GE) crops. The transgene free homozygous mutants with desired genetic modifications at the targeted loci and without RGEN transgene construct could be selected by selfing of $\mathrm{GE}_{0}$ generation plants and after segregation of the transgene in the next $\mathrm{GE}_{1}$ generation. The GE plants could be selected by PCR/RE genotyping and DNA sequencing of clones and negatively selecting for the transgene free plants with desired modification in the first generation only. 
system in plants as this ultimate plant genome editing toolkit shows encouraging potential for producing more complex useful agronomic traits in plant varieties (Quétier, 2016). This technology can better supplement the classical breeding techniques for understanding the complex quantitative traits, whose combinations of genes are selected by traditional breeding methods (Fichtner et al., 2014). The GE crops with the appropriate regulatory structures in place might prove to be more acceptable than the plants that carry foreign DNA in their genomes (Lozano-Juste and Cutler, 2014; Osakabe and Osakabe, 2015). The US Department of Agriculture has indicated that GE plants without any foreign DNA will not be considered as GMOs, however, the European Commission is expected to publish in the near future about the regulatory uncertainty of genome editing (Jones, 2015). The extent of the potential of CRISPR technology in applied plant research and crop breeding benefit for world's food security will depend upon the performance and public perception of the GE crop varieties (Belhaj et al., 2015; Jones, 2015; Wolt et al., 2015).

\section{FUTURE PROSPECTS}

The potential future crops for sustainable productive agriculture by genome editing are those which have better pest resistance, with enhanced nutritional value, and that are able to survive in changing climate. Climate resilient agriculture for combating abiotic and biotic stress is the future of crop improvement using genome editing for both the targeted mutagenesis mediated manipulation and study of transcriptional control by dissection of physiological and molecular cross talk under combined stress (Kissoudis et al., 2014; Jain, 2015). Genome editing will play very important role in developing new bio-energy crops, which could give maximum yield on wastelands and changing climate (Bosch and Hazen, 2013). This technology could offer any possible novel genome-editing concept for plants in order to improve crops for better nutrition and food security. We here suggest some of the possible concepts, which could be utilized for crop improvement and plant biotechnology applications (Liu and Fan, 2014) (Figure 6). Further, direct delivery methods of Cas9 and gRNA using Agrobacterium and Viral replicons by using nanoparticles can be very useful for simplifying the genome editing technology (Hiei et al., 2014; Khatodia et al., 2014; Nonaka and Ezura, 2014). Inducible Cas9 system for transcription modulation like split-Cas9 for chemically inducible system and light activated Cas9 effector (LACE) could be utilized for crop improvement in future (Polstein and Gersbach, 2015; Zetsche et al., 2015). The generation of large-scale whole-genome targeted sgRNA library for high-throughput loss-of-function screening applications based on the CRISPRi system like that of RNAi is particularly feasible for model plants in future (Heintze et al., 2013).

Root trait is very important target for crop improvement using the CRISPR/Cas9 genome editing by studying the regulation of stress responses at cellular level in roots, allele replacement for QTL validation, and the epigenetic regulation of roots (Ahmadi et al., 2014). A major breeding target using CRISPR/Cas9 genome editing is to build cereal crops harboring root systems that can capture unevenly distributed water and nutrient resources in climate instability and resource scarcity. Targeted mutagenesis using the CRISPR-Cas9 system can bring advances in functional genomic research of legumes especially by generating the target mutants of the genes involved in roots and nodules (Sun et al., 2015). A recently published report of naturally transgenic sweet potato having T-DNAs has raised a few questions (Kyndt et al., 2015). The CRISPR/Cas9 editing methods can be used to delete T-DNAs for 'non-transgenic' sweet potato generation to test whether the T-DNAs confer a useful phenotype in naturally transgenic sweet potato (Jones, 2015). This technology can also be utilized for the production of haploid plants by alterations in

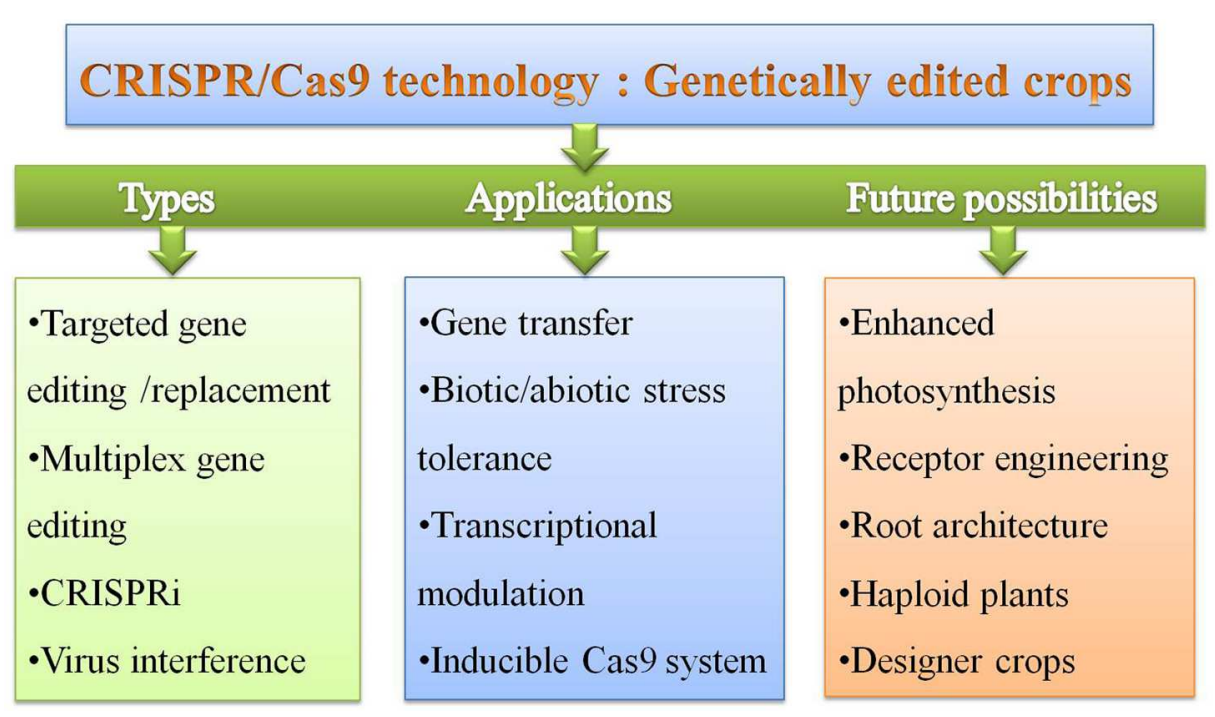

FIGURE 6 | The types, applications and future possibilities of CRISPR/Cas9 system for development of GE crops for crop improvement. 
the histone proteins found in centromeric nucleosomes (Kumar and Jain, 2014). Centromeric histone H3 protein (CENH3) is a member of the kinetochore complex, which is required for kinetochore formation and for chromosome segregation. Using the CRISPR/Cas9 technology, the expression of endogenous CENH3 gene can be suppressed by targeted mutagenesis in a plant, which can lead to selective loss of one set of chromosomes for haploid plants production (Maheswari et al., 2014). The ease and multiplicity of CRISPR/Cas system have shown the potential in three dimensions of plant functional genomics, i.e., genomics, transcriptomics, and epigenomics (Puchta, 2016). This can allow simultaneous induction as well as repression of certain sets of genes and at the same time aid in reprogramming the epigenome (Puchta, 2016).

In conclusion, this technique is getting more precise and efficient day by day, as the future opportunities like inducible Cas9 expression and direct delivery of Cas9 protein, are being explored in different organisms and cell types among the biologists all over the world (Ramakrishna et al., 2014; Polstein and Gersbach, 2015). These novel improvements can help avoid the boosting of off-target effects by expressing the Cas9/sgRNA only when required. Plant biotechnological applications of Cas 9 technology not only lies in engineering non-food crops but

\section{REFERENCES}

Ahmadi, N., Audebert, A., Bennett, M. J., Bishopp, A., de Oliveira, A. C., Courtois, B., et al. (2014). The roots of future rice harvests. Rice (NY) 7, 29. doi: 10.1186/s12284-014-0029-y

Ali, Z., Abulfaraj, A., Idris, A., Ali, S., Tashkandi, M., and Mahfouz, M. M. (2015a). CRISPR/Cas9-mediated viral interference in plants. Genome Biol. 16, 238. doi: 10.1186/s13059-015-0799-6

Ali, Z., Abul-faraj, A., Piatek, M., and Mahfouz, M. M. (2015b). Activity and specificity of TRV-mediated gene editing in plants. Plant Signal. Behav. 10:e1044191. doi: 10.1080/15592324.2015.1044191

Anders, C., Niewoehner, O., Duerst, A., and Jinek, M. (2014). Structural basis of PAM-dependent target DNA recognition by the Cas 9 endonuclease. Nature 513, 569-573. doi: 10.1038/nature13579

Anton, T., Bultmann, S., Leonhardt, H., and Markaki, Y. (2014). Visualization of specific DNA sequences in living mouse embryonic stem cells with a programmable fluorescent CRISPR/Cas system. Nucleus 5, 163-172. doi: $10.4161 /$ nucl. 28488

Araki, M., and Ishii, T. (2015). Towards social acceptance of plant breeding by genome editing. Trends Plant Sci. 20, 145-149. doi: 10.1016/j.tplants.2015.01.010

Baltes, N. J., Gil-Humanes, J., Cermak, T., Atkins, P. A., and Voytas, D. F. (2014). DNA replicons for plant genome engineering. Plant Cell 26, 151-163. doi: 10.1105/tpc.113.119792

Baltes, N. J., Hummel, A. W., Konecna, E., Cegan, R., Bruns, A. N., Bisaro, D. M., et al. (2015). Conferring resistance to geminiviruses with the CRISPR-Cas prokaryotic immune system. Nat. Plants 1, 15145. doi: 10.1038/nplants.2015.145

Barrangou, R. (2013). CRISPR-Cas systems and RNA-guided interference. Wiley Interdiscip. Rev. RNA 4, 267-278. doi: 10.1002/wrna.1159

Belhaj, K., Chaparro-Garcia, A., Kamoun, S., and Nekrasov, V. (2013). Plant genome editing made easy: targeted mutagenesis in model and crop plants using the CRISPR/Cas system. Plant Methods 9, 39. doi: 10.1186/1746-48 11-9-39

Belhaj, K., Chaparro-Garcia, A., Kamoun, S., Patron, N. J., and Nekrasov, V. (2015). Editing plant genomes with CRISPR/Cas9. Curr. Opin. Biotechnol. 32, 76-84. doi: 10.1016/j.copbio.2014.11.007 also have the potential of generating altogether new plant varieties/species for production of specialized chemicals and biomaterials. The swiftness at which Cas9/sgRNA system has developed and improved, it has actually provided human control over genetic information with a huge genomic revolution. This will revolutionize both basic and applied research to improve a wide variety of agronomic traits in crop plants. Another remarkable worth of this CRISPR/cas9 system is that it is by far the most user friendly technique among all the currently available genome editing techniques.

\section{AUTHOR CONTRIBUTIONS}

SK and KB conceived and wrote the manuscript. NP reviewed and helped in figures. SMPK and NT reviewed the manuscript. All authors listed, have made substantial, direct, and intellectual contribution to the work, and approved it for publication.

\section{ACKNOWLEDGMENT}

We thank the reviewers for their many informative comments and suggestions, which have improved the manuscript a lot.

Bortesi, L., and Fischer, R. (2015). The CRISPR/Cas9 system for plant genome editing and beyond. Biotechnol. Adv. 33, 41-52. doi: 10.1016/j.biotechadv.2014.12.006

Bosch, M., and Hazen, S. P. (2013). Lignocellulosic feedstocks: research progress and challenges in optimizing biomass quality and yield. Front. Plant Sci. 4:474. doi: $10.3389 /$ fpls.2013.00474

Brooks, C., Nekrasov, V., Lippman, Z., and Van Eck, J. (2014). Efficient gene editing in tomato in the first generation using the CRISPR/Cas9 system. Plant Physiol. 166, 1292-1297. doi: 10.1104/pp.114.247577

Butler, N. M., Atkins, P. A., Voytas, D. F., and Douches, D. S. (2015). Generation and inheritance of targeted mutations in potato (Solanum tuberosum L.) Using the CRISPR/Cas System. PLoS ONE 10:e0144591. doi: 10.1371/journal.pone.0144591

Cai, Y., Chen, L., Liu, X., Sun, S., Wu, C., Jiang, B., et al. (2015). CRISPR/Cas9mediated genome editing in soybean hairy roots. PLoS ONE 10:e136064. doi: 10.1371/journal.pone.0136064

Cermak, T., Baltes, N. J., Cegan, R., Zhang, Y., and Voytas, D. F. (2015). Highfrequency, precise modification of the tomato genome. Genome Biol. 16, 232. doi: 10.1186/s13059-015-0796-9

Chaparro-Garcia, A., Kamoun, S., and Nekrasov, V. (2015). Boosting plant immunity with CRISPR/Cas. Genome Biol. 16, 254. doi: 10.1186/s13059-0150829-4

Chen, K., and Gao, C. (2014). Targeted genome modification technologies and their applications in crop improvements. Plant Cell Rep. 33, 575-583. doi: 10.1007/s00299-013-1539-6

Cong, L., Ran, F. A., Cox, D., Lin, S., Barretto, R., Habib, N., et al. (2013). Multiplex genome engineering using CRISPR/Cas systems. Science 339, 819-823. doi: $10.1126 /$ science. 1231143

Doudna, J. A., and Charpentier, E. (2014). Genome editing. The new frontier of genome engineering with CRISPR-Cas9. Science 346, 1258096. doi: $10.1126 /$ science. 1258096

Endo, M., Mikami, M., and Toki, S. (2014). Multigene knockout utilizing off-target mutations of the CRISPR/Cas9 system in rice. Plant Cell Physiol. 1, 1-7. doi: 10.1093/pcp/pcu154

Fan, D., Liu, T., Li, C., Jiao, B., Li, S., Hou, Y., et al. (2015). Efficient CRISPR/Cas9mediated targeted mutagenesis in Populus in the first generation. Sci. Rep. 5, 12217. doi: $10.1038 /$ srep 12217 
Fauser, F., Schiml, S., and Puchta, H. (2014). Both CRISPR/Cas-based nucleases and nickases can be used efficiently for genome engineering in Arabidopsis thaliana. Plant J. 79, 348-359. doi: 10.1111/tpj.12554

Feng, Z., Mao, Y., Xu, N., Zhang, B., Wei, P., Yang, D.-L., et al. (2014). Multigeneration analysis reveals the inheritance, specificity, and patterns of CRISPR/Cas-induced gene modifications in Arabidopsis. Proc. Natl. Acad. Sci. U.S.A. 111, 4632-4637. doi: 10.1073/pnas.1400822111

Feng, Z., Zhang, B., Ding, W., Liu, X., Yang, D.-L., Wei, P., et al. (2013). Efficient genome editing in plants using a CRISPR/Cas system. Cell Res. 23, 1229-1232. doi: $10.1038 /$ cr.2013.114

Fichtner, F., Urrea Castellanos, R., and Ülker, B. (2014). Precision genetic modifications: a new era in molecular biology and crop improvement. Planta 239, 921-939. doi: 10.1007/s00425-014-2029-y

Fu, Y., Sander, J. D., Reyon, D., Cascio, V. M., and Joung, J. K. (2014). Improving CRISPR-Cas nuclease specificity using truncated guide RNAs. Nat. Biotechnol. 32, 279-284. doi: $10.1038 /$ nbt.2808

Gaj, T., Gersbach, C. A., and Barbas, C. F. (2013). ZFN, TALEN, and CRISPR/Casbased methods for genome engineering. Trends Biotechnol. 31, 397-405. doi: 10.1016/j.tibtech.2013.04.004

Gao, J., Wang, G., Ma, S., Xie, X., Wu, X., Zhang, X., et al. (2014). CRISPR/Cas9mediated targeted mutagenesis in Nicotiana tabacum. Plant Mol. Biol. 87, 99-110. doi: 10.1007/s11103-014-0263-0

Gao, Y., and Zhao, Y. (2014a). Self-processing of ribozyme-flanked RNAs into guide RNAs in vitro and in vivo for CRISPR-mediated genome editing. J. Integr. Plant Biol. 56, 343-349. doi: 10.1111/jipb.12152

Gao, Y., and Zhao, Y. (2014b). Specific and heritable gene editing in Arabidopsis. Proc. Natl. Acad. Sci. U.S.A. 111, 4357-4358. doi: 10.1073/pnas.1402295111

Giddings, L. V., Potrykus, I., Ammann, K., and Fedoroff, N. V. (2012). Confronting the gordian knot. Nat. Biotechnol. 30, 208-209. doi: 10.1038/nbt.2145

Gilbert, L. A., Horlbeck, M. A., Adamson, B., Villalta, J. E., Chen, Y., Whitehead, E. H., et al. (2015). Genome-scale CRISPR-mediated control of gene repression and activation. Cell 159, 647-661. doi: 10.1016/j.cell.2014.09.029

Gilbert, L. A., Larson, M. H., Morsut, L., Liu, Z., Brar, G. A., Torres, S. E., et al. (2013). CRISPR-mediated modular RNA-guided regulation of transcription in eukaryotes. Cell 154, 442-451. doi: 10.1016/j.cell.2013.06.044

Hartung, F., and Schiemann, J. (2014). Precise plant breeding using new genome editing techniques: opportunities, safety and regulation in the EU. Plant J. 78, 742-752. doi: 10.1111/tpj.12413

Heintze, J., Luft, C., and Ketteler, R. (2013). A CRISPR/Cas for high-throughput silencing. Front. Genet. 4:193. doi: 10.3389/fgene.2013.00193

Hiei, Y., Ishida, Y., and Komari, T. (2014). Progress of cereal transformation technology mediated by Agrobacterium tumefaciens. Front. Plant Sci. 5:628. doi: 10.3389/fpls.2014.00628

Hilbeck, A., Meier, M., Römbke, J., Jänsch, S., Teichmann, H., and Tappeser, B. (2011). Environmental risk assessment of genetically modified plants - concepts and controversies. Environ. Sci. Eur. 23, 1-12. doi: 10.1186/2190-4715-23-13

Hyun, Y., Kim, J., Cho, S. W., Choi, Y., Kim, J.-S., and Coupland, G. (2015). Site-directed mutagenesis in Arabidopsis thaliana using dividing tissue-targeted RGEN of the CRISPR/Cas system to generate heritable null alleles. Planta 241, 271-284. doi: 10.1007/s00425-014-2180-5

Ito, Y., Nishizawa-Yokoi, A., Endo, M., Mikami, M., and Toki, S. (2015). CRISPR/Cas9-mediated mutagenesis of the RIN locus that regulates tomato fruit ripening. Biochem. Biophy. Res. Commun. 467, 76-82. doi: 10.1016/j.bbrc.2015.09.117

Jacobs, T. B., LaFayette, P. R., Schmitz, R. J., and Parrott, W. A. (2015). Targeted genome modifications in soybean with CRISPR/Cas9. BMC Biotechnol. 15:16. doi: 10.1186/s12896-015-0131-2

Jain, M. (2015). Function genomics of abiotic stress tolerance in plants: a CRISPR approach. Front. Plant Sci. 6:375. doi: 10.3389/fpls.2015.00375

Ji, X., Zhang, H., Zhang, Y., Wang, Y., and Gao, C. (2015). Establishing a CRISPRCas-like immune system conferring DNA virus resistance in plants. Nat. Plants 1, 15144. doi: 10.1038/nplants.2015.144

Jia, H., and Wang, N. (2014). Targeted genome editing of sweet orange using Cas9/sgRNA. PLoS ONE 9:e93806. doi: 10.1371/journal.pone.0093806

Jiang, W., Yang, B., and Weeks, D. P. (2014). Efficient CRISPR/Cas9-mediated gene editing in Arabidopsis thaliana and inheritance of modified genes in the T2 and T3 generations. PLoS ONE 9:e99225. doi: 10.1371/journal.pone.0099225
Jiang, W., Zhou, H., Bi, H., Fromm, M., Yang, B., and Weeks, D. P. (2013). Demonstration of CRISPR/Cas9/sgRNA-mediated targeted gene modification in Arabidopsis, tobacco, sorghum and rice. Nucleic Acids Res. 41:e188. doi: 10.1093/nar/gkt780

Jinek, M., East, A., Cheng, A., and Li, S. (2013). RNA-programmed genome editing in human cells. Elife 2:e00471. doi: 10.7554/eLife.00471

Jinek, M., Jiang, F., Taylor, D. W., Sternberg, S. H., Kaya, E., Ma, E., et al. (2014). Structures of Cas9 endonucleases reveal RNA-mediated conformational activation. Science 343:1247997. doi: 10.1126/science.1247997

Johnson, R. A., Gurevich, V., Filler, S., Samach, A., and Levy, A. (2015). Comparative assessments of CRISPR-Cas nucleases' cleavage efficiency in planta. Plant Mol. Biol. 87, 143-156. doi: 10.1007/s11103-014-0266-x

Jones, H. D. (2015). Regulatory uncertainty over genome editing. Nat Plants 1 , 14011. doi: $10.1038 /$ nplants.2014.11

Kanchiswamy, C. N., Sargent, D. J., Velasco, R., Maffei, M. E., and Malnoy, M. (2015). Looking forward to genetically edited fruit crops. Trends Biotechnol. 33, 62-64. doi: 10.1016/j.tibtech.2014.07.003

Khatodia, S., Kharb, P., Batra, P., and Chowdhury, V. K. (2014). Development and characterization of transgenic chickpea (Cicer arietinum L.) plants with cry1Ac gene using tissue culture independent protocol. Int. J. Adv. Res. 2, 323-331.

Khatodia, S., and Khurana, S. M. P. (2014). Trending: the Cas nuclease mediated genome editing technique. Biotech Today 4, 46-49. doi: 10.5958/23220996.2014.00019.2

Kim, H., and Kim, J. S. (2014). A guide to genome engineering with programmable nucleases. Nat. Rev. Genet. 15, 321-334. doi: 10.1038/nrg3686

Kissoudis, C., van de Wiel, C., Visser, R. G. F., and van der Linden, G. (2014). Enhancing crop resilience to combined abiotic and biotic stress through the dissection of physiological and molecular crosstalk. Front. Plant Sci. 5:207. doi: $10.3389 /$ fpls.2014.00207

Kumar, V., and Jain, M. (2014). The CRISPR-Cas system for plant genome editing: advances and opportunities. J. Exp. Bot. 66, 47-57. doi: 10.1093/jxb/eru429

Kyndt, T., Quispe, D., Zhai, H., Jarret, R., Ghislain, M., Liu, Q., et al. (2015). The genome of cultivated sweet potato contains Agrobacterium T-DNAs with expressed genes: an example of a naturally transgenic food crop. Proc. Natl. Acad. Sci. U.S.A. 112, 5844-5849. doi: 10.1073/pnas.14196 85112

Laganà, A., Shasha, D., and Croce, C. M. (2014). Synthetic RNAs for gene regulation: design principles and computational tools. Front. Bioeng. Biotechnol. 2:65. doi: 10.3389/fbioe.2014.00065

Larson, M. H., Gilbert, L. A., Wang, X., Lim, W. A., Weissman, J. S., and Qi, L. S. (2013). CRISPR interference (CRISPRi) for sequence-specific control of gene expression. Nat. Protoc. 8, 2180-2196. doi: 10.1038/nprot.2013.132

Lau, W., Fischbach, M. A., Osbourn, A., and Sattely, E. S. (2014). Key applications of plant metabolic engineering. PLoS Biol. 12:e1001879. doi: 10.1371/journal.pbio.1001879

Li, J. F., Norville, J. E., Aach, J., McCormack, M., Zhang, D., Bush, J., et al. (2013). Multiplex and homologous recombination-mediated genome editing in Arabidopsis and Nicotiana benthamiana using guide RNA and Cas9. Nat. Biotechnol. 31, 688-691. doi: 10.1038/nbt. 2654

Liang, Q., Huashan, L., Yunhan, J., and Chunsheng, D. (2015). The molecular mechanism of CRISPR/Cas9 system and its application in gene therapy of human diseases. Yi Chuan 37, 974-982. doi: 10.16288/j.yczz.15-109

Liang, Z., Zhang, K., Chen, K., and Gao, C. (2014). Targeted mutagenesis in Zea mays using TALENs and the CRISPR/Cas system. J. Genet. Genomics 41, 63-68. doi: 10.1016/j.jgg.2013.12.001

Liu, L., and Fan, X. D. (2014). CRISPR-Cas system: a powerful tool for genome engineering. Plant Mol. Biol. 85, 209-218. doi: 10.1007/s11103-014-0188-7

Liu, W., Yuan, J. S., and Stewart, C. N. (2013). Advanced genetic tools for plant biotechnology. Nat. Rev. Genet. 14, 781-793. doi: 10.1038/nrg3583

Lowder, L. G., Zhang, D., Baltes, N. J., Paul, J. W. III, Tang, X., Zheng, X., et al. (2015). A CRISPR/Cas9 toolbox for multiplexed plant genome editing and transcriptional regulation. Plant Physiol. 169, 971-985. doi: 10.1104/pp.15.00636

Lozano-Juste, J., and Cutler, S. R. (2014). Plant genome engineering in full bloom. Trends Plant Sci. 19, 284-287. doi: 10.1016/j.tplants.2014.02.014 
Lusser, M., Parisi, C., Plan, D., and Rodríguez-Cerezo, E. (2012). Deployment of new biotechnologies in plant breeding. Nat. Biotechnol. 30, 231-239. doi: $10.1038 /$ nbt. 2142

Ma, X., Zhang, Q., Zhu, Q., Liu, W., Chen, Y., Qiu, R., et al. (2015). A robust CRISPR/Cas9 system for convenient, high-efficiency multiplex genome editing in monocot and dicot plants. Mol. Plant 8, 1274-1284. doi: 10.1016/j.molp.2015.04.007

Maheswari, A., Comai, L., and Chan, S. (2014). Generation of Haploid Plants. WO2014110274A2. Washington, DC: U.S. Patent and Trademark Office.

Mahfouz, M. M., Piatek, A., and Stewart, C. N. (2014). Genome engineering via TALENs and CRISPR/Cas9 systems: challenges and perspectives. Plant Biotechnol. J. 12, 1006-1014. doi: 10.1111/pbi.12256

Mali, P., Aach, J., Stranges, P. B., Esvelt, K. M., Moosburner, M., Kosuri, S., et al. (2013). CAS9 transcriptional activators for target specificity screening and paired nickases for cooperative genome engineering. Nat. Biotechnol. 31, 833-838. doi: 10.1038/nbt.2675

Mao, Y., Zhang, H., Xu, N., Zhang, B., Gou, F., and Zhu, J.-K. (2013). Application of the CRISPR-Cas system for efficient genome engineering in plants. Mol. Plant 6, 2008-2011. doi: 10.1093/mp/sst121

Miao, J., Guo, D., Zhang, J., Huang, Q., Qin, G., Zhang, X., et al. (2013). Targeted mutagenesis in rice using CRISPR-Cas system. Cell Res. 23, 1233-1236. doi: $10.1038 / \mathrm{cr} .2013 .123$

Michno, J. M., Wang, X., Liu, J., Curtin, S. J., Kono, T. J., and Stupar, R. M. (2015). CRISPR/Cas mutagenesis of soybean and Medicago truncatula using a new web-tool and a modified Cas9 enzyme. GM Crops Food 6, 243-252. doi: 10.1080/21645698.2015.1106063

Nekrasov, V., Staskawicz, B., Weigel, D., Jones, J. D. G., and Kamoun, S. (2013). Targeted mutagenesis in the model plant Nicotiana benthamiana using Cas 9 RNA-guided endonuclease. Nat. Biotechnol. 31, 691-693. doi: 10.1038/nbt.2655

Nonaka, S., and Ezura, H. (2014). Plant-Agrobacterium interaction mediated by ethylene and super-Agrobacterium conferring efficient gene transfer. Front. Plant Sci. 5:681. doi: 10.3389/fpls.2014.00681

Osakabe, Y., and Osakabe, K. (2015). Genome editing with engineered nucleases in plants. Plant Cell Physiol. 56, 389-400. doi: 10.1093/pcp/pcu170

Piatek, A., Ali, Z., Baazim, H., Li, L., Abulfaraj, A., Al-Shareef, S., et al. (2014). RNA-guided transcriptional regulation in planta via synthetic dCas9-based transcription factors. Plant Biotechnol. J. 13, 578-589. doi: 10.1111/pbi.12284

Podevin, N., Devos, Y., Davies, H. V., and Nielsen, K. M. (2012). Transgenic or not? No simple answer! New biotechnology-based plant breeding techniques and the regulatory landscape. EMBO Rep. 13, 1057-1061. doi: 10.1038/embor.2012.168

Polstein, L. R., and Gersbach, C. A. (2015). A light-inducible CRISPR-Cas9 system for control of endogenous gene activation. Nat. Chem. Biol. 11, 198-200. doi: $10.1038 /$ nchembio. 1753

Puchta, H. (2016). Using CRISPR/Cas in three dimensions: towards synthetic plant genomes, transcriptomes and epigenomes. Plant J. doi: 10.1111/tpj.13100 [Epub ahead of print].

Qi, L. S., Larson, M. H., Gilbert, L. A., Doudna, J. A., Weissman, J. S., Arkin, A. P., et al. (2013). Repurposing CRISPR as an RNA-guided platform for sequence-specific control of gene expression. Cell 152, 1173-1183. doi: 10.1016/j.cell.2013.02.022

Quétier, F. (2016). The CRISPR-Cas9 technology: closer to the ultimate toolkit for targeted genome editing. Plant Sci. 242, 65-76. doi: 10.1016/j.plantsci.2015.09.003

Ramakrishna, S., Kwaku Dad, A. B., Beloor, J., Gopalappa, R., Lee, S. K., and Kim, H. (2014). Gene disruption by cell-penetrating peptide-mediated delivery of Cas9 protein and guide RNA. Genome Res. 24, 1020-1027. doi: 10.1101/gr.171264.113

Ran, F. A., Hsu, P. D., Lin, C.-Y., Gootenberg, J. S., Konermann, S., Trevino, A. E., et al. (2013). Double nicking by RNA-guided CRISPR Cas9 for enhanced genome editing specificity. Cell 154, 1380-1389. doi: 10.1016/j.cell.2013. 08.021

Ron, M., Kajala, K., Pauluzzi, G., Wang, D., Reynoso, M. A., Zumstein, K., et al. (2014). Hairy root transformation using Agrobacterium rhizogenes as a tool for exploring cell type-specific gene expression and function using tomato as a model. Plant Physiol. 166, 455-469. doi: 10.1104/pp.114.239392

Saika, H., Nishizawa-Yokoi, A., and Toki, S. (2014). The non-homologous endjoining pathway is involved in stable transformation in rice. Front. Plant Sci. 5:560. doi: $10.3389 /$ fpls. 2014.00560
Sander, J. D., and Joung, J. K. (2014). CRISPR-Cas systems for editing, regulating and targeting genomes. Nat. Biotechnol. 32, 347-355. doi: 10.1038/nbt.2842

Schaart, J. G., van de Wiel, C. C. M., Lotz, L. A. P., and Smulders, M. J. M. (2015). Opportunities for products of new plant breeding techniques. Trends Plant Sci. doi: 10.1016/j.tplants.2015.11.006 [Epub ahead of print].

Schiml, S., Fauser, F., and Puchta, H. (2014). The CRISPR/Cas system can be used as nuclease for in planta gene targeting and as paired nickases for directed mutagenesis in Arabidopsis resulting in heritable progeny. Plant J. 80, 1139-1150. doi: 10.1111/tpj.12704

Shan, Q., Wang, Y., Li, J., Zhang, Y., Chen, K., Liang, Z., et al. (2013). Targeted genome modification of crop plants using a CRISPR-Cas system. Nat. Biotechnol. 31, 686-688. doi: 10.1038/nbt.2650

Shimatani, Z., Nishizawa-Yokoi, A., Endo, M., Toki, S., and Terada, R. (2015). Positive-negative-selection-mediated gene targeting in rice. Front. Plant Sci. 5:748. doi: 10.3389/fpls.2014.00748

Shuman, S., and Glickman, M. S. (2007). Bacterial DNA repair by non-homologous end joining. Nat. Rev. Microbiol. 5, 852-861. doi: 10.1038/nrmicro1768

Sugano, S. S., Shirakawa, M., Takagi, J., Matsuda, Y., Shimada, T., HaraNishimura, I., et al. (2014). CRISPR/Cas9-mediated targeted mutagenesis in the liverwort Marchantia polymorpha L. Plant Cell Physiol. 55, 475-481. doi: $10.1093 / \mathrm{pcp} / \mathrm{pcu} 014$

Sun, X., Hu, Z., Chen, R., Jiang, Q., Song, G., Zhang, H., et al. (2015). Targeted mutagenesis in soybean using the CRISPR-Cas9 system. Sci. Rep. 5, 10342. doi: $10.1038 /$ srep 10342

Svitashev, S., Young, J. K., Schwartz, C., Gao, H., Falco, S. C., and Cigan, A. M. (2015). Targeted mutagenesis, precise gene editing, and site-specific gene insertion in maize using Cas9 and guide RNA. Plant Physiol. 169, 931-945. doi: 10.1104/pp.15.00793

Tanenbaum, M. E., Gilbert, L. A., Qi, L. S., Weissman, J. S., and Vale, R. D. (2014). A protein-tagging system for signal amplification in gene expression and fluorescence imaging. Cell 159, 635-646. doi: 10.1016/j.cell.2014.09.039

Tingting, L., Di, F., Lingyu, R., Yuanzhong, J., Rui, L., and Keming, L. (2015). Highly efficient CRISPR/Cas9-mediated targeted mutagenesis of multiple genes in Populus. Yi Chuan 37, 1044-1052. doi: 10.16288/j.yczz.15-303

Tsai, S. Q., Wyvekens, N., Khayter, C., Foden, J. A., Thapar, V., Reyon, D., et al. (2014). Dimeric CRISPR RNA-guided FokI nucleases for highly specific genome editing. Nat. Biotechnol. 32, 569-576. doi: 10.1038/nbt.2908

Upadhyay, S. K., Kumar, J., Alok, A., and Tuli, R. (2013). RNA-guided genome editing for target gene mutations in wheat. G3 (Bethesda) 3, 2233-2238. doi: 10.1534/g3.113.008847

Voytas, D. F., and Gao, C. (2014). Precision genome engineering and agriculture: opportunities and regulatory challenges. PLoS Biol. 12:e1001877. doi: 10.1371/journal.pbio.1001877

Wang, S., Zhang, S., Wang, W., Xiong, X., Meng, F., and Cui, X. (2015). Efficient targeted mutagenesis in potato by the CRISPR/Cas9 system. Plant Cell Rep. 34, 1473-1476. doi: 10.1007/s00299-015-1816-7

Wang, Y., Cheng, X., Shan, Q., Zhang, Y., Liu, J., Gao, C., et al. (2014). Simultaneous editing of three homoeoalleles in hexaploid bread wheat confers heritable resistance to powdery mildew. Nat. Biotechnol. 32, 947-951. doi: 10.1038/nbt.2969

Wolt, J. D., Wang, K., and Yang, B. (2015). The regulatory status of genome-edited crops. Plant Biotechnol. J. 14, 510-518. doi: 10.1111/pbi.12444

Woo, J. W., Kim, J., Kwon, S. I., Corvalan, C., Cho, S. W., Kim, H., et al. (2015). DNA-free genome editing in plants with preassembled CRISPR-Cas9 ribonucleoproteins. Nat. Biotechnol. 33, 1162-1164. doi: 10.1038/nbt.3389

Wyman, C., and Kanaar, R. (2006). DNA double-strand break repair: all's well that ends well. Annu. Rev. Genet. 40, 363-383. doi: 10.1146/annurev.genet.40.110405.090451

Xie, K., Minkenberg, B., and Yang, Y. (2015). Boosting CRISPR/Cas9 multiplex editing capability with the endogenous tRNA-processing system. Proc. Natl. Acad. Sci. U.S.A. 112, 3570-3575. doi: 10.1073/pnas.1420294112

Xie, K., and Yang, Y. (2013). RNA-guided genome editing in plants using a CRISPR-Cas system. Mol. Plant 6, 1975-1983. doi: 10.1093/mp/sst119

Xing, H.-L., Dong, L., Wang, Z.-P., Zhang, H.-Y., Han, C.-Y., Liu, B., et al. (2014). A CRISPR/Cas9 toolkit for multiplex genome editing in plants. BMC Plant Biol. 14:327. doi: 10.1186/s12870-014-0327-y

Xu, R. F., Li, H., Qin, R. Y., Li, J., Qiu, C. H., Yang, Y. C., et al. (2015). Generation of inheritable and "transgene clean" targeted genome-modified rice 
in later generations using the CRISPR/Cas9 system. Sci. Rep. 5, 11491. doi: $10.1038 /$ srep 11491

Xu, T., Li, Y., Van Nostrand, J. D., He, Z., and Zhou, J. (2014). Cas9-based tools for targeted genome editing and transcriptional control. Appl. Environ. Microbiol. 80, 1544-1552. doi: 10.1128/AEM.03786-13

Yin, K., Han, T., Liu, G., Chen, T., Wang, Y., Yu, A. Y., et al. (2015). A geminivirusbased guide RNA delivery system for CRISPR/Cas 9 mediated plant genome editing. Sci. Rep. 5, 14926. doi: 10.1038/srep14926

Zetsche, B., Volz, S. E., and Zhang, F. (2015). A split-Cas9 architecture for inducible genome editing and transcription modulation. Nat. Biotechnol. 33, 139-142. doi: $10.1038 /$ nbt.3149

Zhang, H., Zhang, J., Wei, P., Zhang, B., Gou, F., Feng, Z., et al. (2014). The CRISPR/Cas9 system produces specific and homozygous targeted gene editing in rice in one generation. Plant Biotechnol. J. 12, 797-807. doi: $10.1111 /$ pbi. 12200

Zhang, L., and Zhou, Q. (2014). CRISPR/Cas technology: a revolutionary approach for genome engineering. Sci. China Life Sci. 57, 639-640. doi: 10.1007/s11427014-4670-X

Zhang, Z., Mao, Y., Ha, S., Liu, W., Botella, J. R., and Zhu, J. K. (2015). A multiplex CRISPR/Cas9 platform for fast and efficient editing of multiple genes in Arabidopsis. Plant Cell Rep. 1-5. doi: 10.1007/s00299-015-1900-Z
Zhou, H., Liu, B., Weeks, D. P., Spalding, M. H., and Yang, B. (2014). Large chromosomal deletions and heritable small genetic changes induced by CRISPR/Cas9 in rice. Nucleic Acids Res. 42, 10903-10914. doi: $10.1093 / \mathrm{nar} / \mathrm{gku} 806$

Zhou, X., Jacobs, T. B., Xue, L.-J., Harding, S. A., and Tsai, C. J. (2015). Exploiting SNPs for biallelic CRISPR mutations in the outcrossing woody perennial Populus reveals 4-coumarate: CoA ligase specificity and redundancy. New Phytol. 208, 298-301. doi: 10.1111/nph. 13470

Conflict of Interest Statement: The authors declare that the research was conducted in the absence of any commercial or financial relationships that could be construed as a potential conflict of interest.

Copyright (C) 2016 Khatodia, Bhatotia, Passricha, Khurana and Tuteja. This is an open-access article distributed under the terms of the Creative Commons Attribution License (CC BY). The use, distribution or reproduction in other forums is permitted, provided the original author(s) or licensor are credited and that the original publication in this journal is cited, in accordance with accepted academic practice. No use, distribution or reproduction is permitted which does not comply with these terms. 